\title{
EDUCACIÓN A DISTANCIA Y LA COMUNIDAD DE PAÍSES DE LENGUA PORTUGUESA
}

\section{Distance education and the Community of the Country of Portuguese Language}

\author{
Fernanda Maria Melo Alves ${ }^{1}$ \\ José Antonio Moreiro González ${ }^{2}$
}

\begin{abstract}
Resumo: Se hace una reflexión acerca de la relación entre la Educación a Distancia y la Comunidad de Países de Lengua Portuguesa. Se emplea una investigación de carácter descriptivo, exploratorio y cualitativo. A partir de una revisión bibliográfica y documental sobre las Nuevas Tecnologías de la Información y de la Comunicación y la Educación a Distancia en distintas bases de datos bibliográficas, se efectúa la búsqueda de información relativa a la cooperación educativa entre los países de la Comunidad de Países de Lengua Portuguesa, empleando su la web institucional. Seleccionada la literatura apropiada al objeto de estudio, se realiza una lectura analítica de su contenido y se sistematiza la información relativa al problema de investigación. Los resultados plantean dos iniciativas de cooperación educativa paradigmáticas, efectuadas por la Universidade Aberta de Portugal y la Universidade Aberta do Brasil, obtenidas a través de proyectos de cooperación bilaterales, y resaltan la necesidad de implementar la educación a distancia en los países de la Comunidad de Países de Lengua Portuguesa, a través de políticas educativas concertadas para formar ciudadanos críticos y colaborativos.
\end{abstract}

Palavras-chave: Educación a distancia. Comunidad de Paises de Lengua Portuguesa. Cooperación internacional. Desarrollo.

\begin{abstract}
A reflection is made about the relationship between Distance Education and the Community of Portuguese Language Countries. Descriptive, exploratory and qualitative research is used. From a bibliographic and documentary review on the New Information and Communication Technologies and Distance Education in different bibliographic databases, the search for information on educational cooperation between the countries of the Community of Portuguese Language Countries is carried out, using its institutional website. Once the appropriate literature has been selected for the object of study, an analytical reading of its content is carried out and the information related to the research problem is systematized. The results propose two paradigmatic educational cooperation initiatives, carried out by the Universidade Aberta de Portugal and the Universidade Aberta do Brasil, obtained through bilateral cooperation projects. The results propose two paradigmatic educational cooperation initiatives, carried out by the Universidade Aberta of Portugal and the Universidade Aberta of Brasil, obtained through bilateral cooperation projects, and highlight the need to implement distance education in the countries of the Community of Portuguese Language Countries, through concerted educational policies to train critical and collaborative citizens.

Keywords: Long distance education. Community of Portuguese Language Countries. Social inclusion. Development. International cooperation.
\end{abstract}

\footnotetext{
1 Doutora em Documentação: Arquivos e Bibliotecas em entorno digital pela Universidade Carlos III de Madrid. http://lattes.cnpq.br/6468376220858021. Orcid: https://orcid.org/0000-0002-8396$\underline{4053}$.
}

2 Doutor em História pela Universidade Nacional de Educação à Distancia -UNED/Espanha. Lattes: http://lattes.cnpq.br/3656042126236028. Orcid: https://orcid.org/0000-0002-8827-158X. 


\section{Introdución}

La educación es un derecho humano y una fuerza para el desarrollo sostenible y la paz. El derecho a la educación es un pilar de la ciudadanía y la dignidad de las personas, que está consagrado en los tratados internacionales y en la mayoría de las constituciones nacionales.

En las últimas décadas, el uso de las tecnologías de la comunicación (TIC) y la Educación a Distancia (EaD) han suscitado el interés de todos los intervinientes en el proceso educativo y de la sociedad en general, debido a su avance continuado y por el incremento, las nuevas oportunidades y los desafíos ofrecidos por esta modalidad de enseñanza y formación.

La Agenda 2030 sobre el Desarrollo Sostenible (UN, 2005) es un plan de acción a favor de las personas, el planeta y la prosperidad, que tiene la intención de fortalecer la paz universal y el acceso a la justicia. Cada uno de sus objetivos exige el acceso a la educación para capacitar a las personas con los conocimientos, habilidades y valores que las permitan vivir con dignidad, construir sus vidas y contribuir positivamente a su sociedad. La educación se plantea esencialmente en el objetivo 4 (ODS 4), "garantizar una educación de calidad equitativa e inclusiva, y promover oportunidades de aprendizaje permanente para todos" para 2030.

De ahí la importancia en este estudio de establecer reflexiones teóricas y prácticas acerca de la relación entre la EaD y la Comunidad de Países de Lengua Portuguesa (CPLP), que fundamenten nuevas aplicaciones de las TIC en EaD, con el propósito de incrementar nuevos saberes y prácticas educativas, por lo que requiere siempre la necesaria contextualización, teniendo en cuenta la educación a lo largo de la vida y las estrategias mundiales para el siglo XXI.

\section{La educación superior y a distancia: cambios y desafíos}

La responsabilidad principal de garantizar el derecho a una educación de calidad es un compromiso universal y colectivo que requiere voluntad política, colaboración global y regional, junto a la participación de todos los gobiernos, la sociedad civil, el sector privado y las agencias multilaterales, para abordar los desafíos educativos y construir sistemas que sean inclusivos, equitativos y relevantes para todos los estudiantes.

El cambio de siglo ha venido acompañado de múltiples cambios en lo tocante a la educación superior. Entre otros, se destacan algunos marcos significativos que plasman el análisis y la toma de decisiones para lograr las reformas necesarias, como la World Conference on Higher Education in the Twenty-first Century: Vision and Action (UNESCO, 1998) celebrada en Paris. O las Directrices UNESCO/OCDE sobre Calidad en la educación superior transfronteriza (UNESCO, 2005), publicadas por la UNESCO Division of Higher Education. También la Sección de Educación para la reforma, innovación y garantía de calidad (RIQ) del 2005 que, para mejorar la calidad de la educación superior

Rev. Nova Paideia - Revista Interdisciplinar em Educação e Pesquisa Brasília/DF, v. 2, n. 1. p. 35 - 46 - ANO 2020 
en el mundo, aboga por una mayor movilidad de personas, programas e instituciones a través de las fronteras instituciones nacionales, así como la participación del sector privado en la provisión de la educación superior. A estos marcos y eventos se seguirán otros por todo el mundo, que no es preciso enumerar por no ser objeto de este análisis.

Para la comprensión del concepto de educación Coldeway (2011) parte de las variables, de tiempo y espacio, cuya combinación permite cuatro enfoques: 1) la educación en el mismo tiempo y espacio (tradicional), 2) la educación en tiempos diferentes y en el mismo espacio, 3) a educación en el mismo tiempo y en diferentes espacios (chat desde diferentes ubicaciones), 4) en tiempos y espacios diferentes (a sincronía total: un foro, por ejemplo).

Cuando la educación tradicional no puede responder a las exigencias de un contexto particular, es necesario buscar las estrategias educativas para que las personas que habitan en aquellas circunstancias puedan acceder a ellos, por lo que "no es sorprendente que el aprendizaje abierto y a distancia sea considerado cada vez más como una estrategia valiosa para resolver los problemas relacionados con el acceso, la calidad e igualdad en la educación" (UNESCO, 2003 2).

En el presente estudio, se toma como referencia la definición educación a distancia o aprendizaje abierto aceptada por la UNESCO, que hace referencia a cualquier proceso educativo en el que toda o la mayor parte de la enseñanza es llevada a cabo por alguien que no comparte el mismo tiempo y/o espacio que el alumno, por lo que toda o la mayor parte de la comunicación entre profesores y alumnos se desarrolla a través de un medio artificial, sea electrónico o impreso (UNESCO, 2002).

Las raíces de la educación a distancia se remontan más de 200 años atrás. Ha pasado básicamente por tres etapas: 1) estudio por correspondencia, 2) incursión de las universidades e 3) incorporación de la educación a distancia en la mayoría de las formas de educación. Además se ha vuelto inseparable del uso de las TIC para realizar el proceso de mediación. A partir del momento de fusión tecnológica se comienza a hablar de e-learning, pues las TIC hicieron disminuir las barreras de espacio y tiempo y ampliaron las posibilidades de interacción entre los estudiantes y los docentes, los estudiantes entre sí, y los estudiantes respecto a una gran cantidad de objetos de contenido en diferentes formatos (García-Aretio, 1999, p. 12).

Rojas-Machado et al. (2014) al profundizar en los modelos de educación no tradicional, acrecienta los siguientes tipos de educación a distancia:

- La educación a distancia (per se), proceso formativo que se fortalece fundamentalmente con materiales físicos emitidos por la institución educativa que entrega a los estudiantes para el desarrollo del curso (separatas, libros, material multimedia en CD u otro). Suelen ser entregados directamente al alumno o enviados por correo postal, pudiendo existir otras variantes de envío o entrega.

- La educación virtual que se afianza a partir de los dispositivos de internet; lo que

Rev. Nova Paideia - Revista Interdisciplinar em Educação e Pesquisa Brasília/DF, v. 2, n. 1. p. 35 - 46 - ANO 2020 
presupone la utilización y el intercambio de información entre el docente y los estudiantes de manera virtual, ya sea por correo electrónico o mediante plataformas específicamente creadas para esta finalidad, a través de las cuales los alumnos descargan los materiales de clase, suben trabajos o asignaciones; e incluso trabajan de manera colaborativa con sus colegas de clase.

- La educación on-line que tiene como base la educación virtual, pero que le añade su ejecución en tiempo real. De modo que los estudiantes asisten a las clases en vivo, por lo que pueden interactuar con profesores y compañeros, asistir a reuniones de estudio en concurrencia con los colegas, además de asistir a videoconferencias, someterse a evaluaciones en vivo, participar en foros de discusión, desarrollar trabajos grupales y otras tantas.

Sobre cómo organizar un modelo de aprendizaje de educación virtual, y teniendo en vista el entorno de los países de la CPLP, seguimos a Salinas (2004), para quién dicho modelo requiere ciertos componentes: 1) una función pedagógica, referida a actividades y situaciones de enseñanza, materiales de instrucción, apoyo, tutoría y evaluación; 2) una función tecnológica apropiada a las herramientas seleccionadas en conexión con el modelo pedagógico; y 3) una función organizativa del espacio, calendario y gestión de la comunidad. Además, considera ser imprescindibles los siguientes elementos: 1) la comunicación mediada por ordenador (componente tecnológico); 2) los medios didácticos; 3) la flexibilidad (elementos del aprendizaje abierto); 4) el entorno organizativo (componente institucional); 5) el aprendizaje y tutoría (componente didáctico).

A propósito de los resultados exitosos alcanzados en proyectos de cooperación internacional universitaria, Bolaños, Bueno y Alves (2013) platean algunas conclusiones en torno a la modalidad de la enseñanza a distancia, a saber:

- Permite la deslocalización del conocimiento, lo que equipara las oportunidades con independencia del lugar de residencia.

- Suministra información a los alumnos, cuya versión impresa sería imposible de difundir, y hacerlo con independencia del espacio y el tiempo en el que se encuentren profesores y estudiantes.

- Potencia la autonomía de los alumnos y propicia una formación adecuada a sus necesidades personales.

- Favorece la comunicación e interactividad con el profesor, entre los alumnos, y de ambos con la información-documentación.

- Facilita la actualización de los contenidos documentales.

- Ayuda a emplear los materiales y los objetos de aprendizaje, y permite que en los servidores quede registrada la actividad realizada por los estudiantes.

- En fin, ahorra costes, desplazamientos, tiempo y esfuerzos.

Siempre, cualquier avance notable en la tecnología aplicada a los procesos de la información ha cambiado las técnicas de su gestión y organización. Y se hace a partir de las ventajas que esa tecnología ofrece en sí misma (Moreiro, 2018).

Rev. Nova Paideia - Revista Interdisciplinar em Educação e Pesquisa Brasília/DF, v. 2, n. 1. p. 35 - 46 - ANO 2020 


\section{Método}

Para conocer la relación entre la Educación a Distancia y la Comunidad de Países de Lengua Portuguesa (CPLP), se dispuso una investigación descriptiva, identificando las características de la EaD y la CPLP, exploratoria, al explicitarlas y articularlas entre sí, y cualitativa, buscando conseguir, a través de datos, comprender los comportamientos de grupos de personas y organizaciones intervinientes en dichos fenómenos. Parte de la búsqueda de información bibliográfica sobre los Tecnologías de la Información y de la Comunicación para la Educación a Distancia en Google Scholar: desde allí se accedió a las bases de datos bibliográficas Web of Science, Scopus, SCIELO y DIALNET. Luego se efectuó la búsqueda de información relativa a la cooperación educativa entre los países de la CPLP, en su la web institucional. Seleccionada la literatura apropiada, se hizo una lectura analítica de su contenido y se sistematizó la información que se había obtenido como respuesta al problema de investigación. Al ser aún reciente la cooperación en educación a distancia entre los miembros de la CPLP, los resultados resaltan la necesidad de su implementación, a través de políticas y proyectos concertados para formar ciudadanos críticos y colaborativos.

\section{La educación a distancia en los países de la Comunidad de Países de Lengua Portuguesa (CPLP): cooperación para la igualdad y el desarrollo}

La Comunidad de Países de Lengua Portuguesa (CPLP) es un foro multilateral entre países de todo el mundo, cuya lengua es el portugués como idioma oficial o cooficial. Comprende alrededor de 223 millones de habitantes, ubicados en todos los continentes, totalizando un área aproximada de 10.708.674 kilómetros cuadrados. Su sede se ubica en Lisboa, Portugal.

La organización se formó en 1996 con siete estados (Angola, Brasil, Cabo Verde, Guinea-Bissau, Guinea Ecuatorial, Mozambique, Portugal y Santo Tomé y Príncipe). Timor Oriental se unió en el 2002 y Guinea Ecuatorial se incorporó como miembro pleno en 2014. Actualmente, su estructura abarca diecinueve países como miembros observadores, Mauricio, Senegal, Georgia, Japón, Namibia, Turquía, Eslovaquia, Hungría, República Checa, Uruguay, Argentina, Chile, Francia, Andorra, Italia, Luxemburgo, Reino Unido y Serbia. Cerca de una decena de países han presentado su candidatura a la organización. Además de los países miembros y de los observadores, cerca de cincuenta organizaciones de la sociedad civil de los países miembros actúan como observadores consultivos.

Los objetivos generales de la CPLP son: 1) la consulta política y diplomática entre sus estados miembros, a saber, para fortalecer su presencia en el escenario internacional; 2) la cooperación en todos los campos, incluyendo educación, salud, ciencia y tecnología, defensa, agricultura, administración pública, comunicaciones,

Rev. Nova Paideia - Revista Interdisciplinar em Educação e Pesquisa Brasília/DF, v. 2, n. 1. p. 35 - 46 - ANO 2020 
justicia, seguridad pública, cultura, deporte y medios de comunicación; 3) la materialización de proyectos para promover y difundir el idioma portugués.

Los países miembros de la CPLP cooperan en distintas áreas del conocimiento, siendo que la cooperación multilateral en el campo de la educación juega un papel importante. Su Declaración constitutiva (CPLP, 1996) tiene como objetivo "impulsar y profundizar la cooperación en el ámbito universitario, en la formación profesional y en los diversos sectores de la investigación científica y tecnológica para una mayor apreciación de sus recursos humanos y naturales, así como para promover y fortalecer políticas de capacitación del personal".

Desde las últimas décadas del siglo XX, cada país miembro de la CPLP ya tenía experiencia en educación a distancia, obtenida a través de proyectos de cooperación bilaterales. Entre las distintas iniciativas de cooperación educativa se destacan las dos paradigmáticas efectuadas por la Universidade Aberta de Portugal y la Universidade Aberta do Brasil, que pasamos a comentar.

Fundada en 1988, la Universidade Aberta (UAb) es la única institución pública de educación a distancia en Portugal. Debido a su vocación y naturaleza, la institución utiliza en sus actividades de enseñanza, las metodologías y tecnologías más avanzadas de aprendizaje a distancia, orientadas a la educación sin fronteras geográficas o barreras físicas, prestando especial atención a la expansión de la lengua y la cultura portuguesas en los países y comunidades de lengua portuguesa.

La cooperación de la UAb en educación sin fronteras o educación transnacional denota una realidad en la que la oferta educativa, respondiendo a la globalización del mercado, va más allá de los límites convencionales, ya sean geográficos, espaciales o temporales. En ese sentido, se formó la Red Global de Aprendizaje para el Desarrollo (GDLN), Red Lusófona, cuyo objetivo es unir a los países de lengua portuguesa a través de centros de educación a distancia para permitir el intercambio de conocimientos y experiencias desde/hacia el desarrollo.

La GDLN está compuesta por una red global interactiva de Centros de Educación a Distancia (CEAD), conectados a través de comunicaciones rápidas y efectivas (IP, RDSI), equipados con plataformas y equipos TIC destinados al intercambio de conocimientos y experiencias, cuyas principales funciones son las siguientes:

- Promoción del diseño para poner en marcha el proyecto.

- Reuniones de trabajo, coordinación, capacitación del personal operativo.

- Desarrollo de capacidades en proyectos de desarrollo, preparación de misiones y comunidades de profesionales.

- Apoyo para la formación de comunidades de expertos.

- Intercambio de resultados y experiencias entre compañeros. 
- Medio para la realización de conferencias, eventos formales, lanzamiento de publicaciones, conferencias de prensa (audiencias amplias y dispersas).

- Apoyo en situaciones de crisis, cuando no se recomienda viajar (conflictos armados, pandemias, etc.).

El GDLN comenzó en 2002 con dos proyecto, la cooperación entre la Universidad Abierta (UAb), el Centro de Educación a Distancia Timor-Leste (CEAD-Díli) y el Instituto de Administración Nacional (INA) de Portugal, para formar funcionarios públicos de la administración timorense, seguido del Proyecto Plurianual, con el apoyo de la Cooperación Portuguesa (IPAD), en colaboración con la Universidad Agostinho Neto de Angola (UAN) y la Universidade Aberta de Portugal (UAb) para instalar un centro de educación a distancia en la UAN, mejorando el centro correspondiente de UAb, apoyar el funcionamiento de ambos y estimular el intercambio de conocimientos entre países de lengua portuguesa (PLP). Las actividades de dicha red se están ampliando al resto de países lusófonos, a través de proyectos adecuados al contexto de cada uno.

El sistema de la Universidad Abierta de Brasil (UAB) se creó en 2005 mediante el establecimiento de una asociación de los tres niveles de administración pública (federal, estatal y municipal) con instituciones públicas de educación superior y otros agentes educativos interesados en ofrecer cursos de educación superior a distancia. Vino a ampliar la oferta de cursos y programas de educación superior en el país.

Para maximizar los beneficios del sistema brasileño de la UAB y atender a las necesidades de cooperación internacional, se optó por la internacionalización de sus servicios a través del Programa de la Universidad Abierta de Brasil (UAB)/MozambiqueExpansión de Educación superior a distancia en la República de Mozambique, establecido en 2010 para poner en marcha un sistema de cooperación entre los gobierno de ambos países. En el proyecto participaron seis instituciones de duración superior, de las que cuatro eran brasileñas (UFG, UFF, UNIRIO e UFJF) y dos mozambiqueñas (Universidade Pedagógica e Eduardo Mondlane). Se ofrecieron cuatro cursos de pregrado, con doble certificación:

- Administración pública: impartido por la Universidad Federal de Juiz de Fora (UFJF) con la Universidad Eduardo Mondlane (UEM).

- Ciencias biológicas: ofrecido por la Universidad Federal de Goiás (UFG) con el Universidad Pedagógica (UP).

- Matemáticas: colaboración entre la Universidade Federal Fluminense (UFF) con el Universidad Pedagógica (UP).

- Educación básica: ofrecida por UNIRIO con la Universidad Pedagógica (UP).

Según Souza $(2017,2018)$, participante en el proyecto, se reunió información de varias publicaciones sobre los resultados alcanzados. Se detectaron dificultades para implantar el programa en su conjunto, en especial la falta de equipos de Internet para las clases, el material didáctico inadecuado y el pago tardío de becas. Y aunque la CAPES

Rev. Nova Paideia - Revista Interdisciplinar em Educação e Pesquisa Brasília/DF, v. 2, n. 1. p. 35 - 46 - ANO 2020 
desactivó el Programa en 2015, los distintos participantes consideraron la experiencia relevante y que, mejor planificada, debería reanudarse. Conscientes de los resultados exitosos de decenas de proyectos de cooperación internacional en educación a distancia y de las ventajas de una cooperación concertada, expertos y autoridades de la CPLP decidieron definir políticas y proyectos comunes.

El evento "Educación a distancia en la CPLP" de 2018, estuvo organizado por la Universidade Aberta (Portugal) e incluyó entre otras actividades, la ceremonia de creación de la Asociación de Educación a Distancia de Países de Lengua Portuguesa, integrando la Universidad Agostinho Neto de Angola, la Coordinación del Personal de Educación Superior de la Brasil (CAPES), la Universidad de Cabo Verde, la Universidad Nacional de Guinea Ecuatorial, la Universidad Eduardo Mondlane de Mozambique, la Universidad Abierta de Portugal, la Universidad de Santo Tomé y Príncipe, y la Universidad Nacional de Timor-Leste.

En el momento de su creación, se definieran diversos objetivos para la Asociación de Educación a Distancia de los Países de Lengua Portuguesa:

- Contribuir a la creación de un espacio para la Ciencia, Tecnología y Educación Superior de CPLP, de acuerdo con los objetivos definidos, a través de la educación a distancia y en red.

- Participar en programas de cualificación para agentes en diferentes niveles de educación en el área común de CPLP.

- Capacitar a los académicos y profesionales en general para el uso masivo y efectivo de las metodologías de educación a distancia y en red más avanzadas.

- Fomentar el desarrollo permanente de metodologías de educación a distancia y en red para fines comunes.

- Promover el desarrollo acelerado y sostenido de la oferta de programas de educación y capacitación a distancia y en red para residentes en países de lengua portuguesa, así como para miembros en general de la CPLP en el mundo, con énfasis en las diásporas, de acuerdo con las necesidades verificadas.

- Fomentar la creación de asociaciones estratégicas entre instituciones, públicas y privadas, de países de lengua portuguesa.

- Contribuir activamente al aumento de la alfabetización digital para los hablantes de portugués en el mundo, abriendo puertas a su inserción social y profesional en la sociedad digital.

- Fomentar la creación de plataformas virtuales para compartir buenas prácticas, modelos y métodos de aprendizaje, así como principios organizativos en educación a distancia y en red, que promuevan la calidad de los servicios prestados.

- Aumentar y racionalizar el intercambio de experiencias e información entre las instituciones y los individuos que trabajan en educación a distancia y en red en países de lengua portuguesa. 
- Promover la conexión de las instituciones asociadas con las sociedades de los países y de la CPLP en el mundo, para unir a las personas, mejorar el conocimiento mutuo y el gusto por el aprendizaje y el conocimiento.

- Llevar a cabo iniciativas conjuntas en los campos científico, tecnológico y de intercambio de conocimientos.

- Fomentar y apoyar el aumento de la movilidad, física y virtual, de docentes, investigadores, técnicos, estudiantes y aprendices en general.

- Valorar la creación de conocimiento en ciencia, cultura, artes y tecnología en portugués.

- Aumentar los niveles de transferencia de conocimiento en investigación y educación a distancia y en red entre socios.

- Ampliar la red de entidades asociadas, independientemente de su naturaleza legal, y monitorear los resultados de la cooperación.

- Realizar estudios sobre educación a distancia y en red en la CPLP y apoyar la creación de bases de datos estadísticas.

- Contribuir al reconocimiento de títulos y calificaciones obtenidas en la modalidad de educación a distancia y en red en CPLP.

La Asociación de Educación a Distancia de Países de Lengua Portuguesa trabaja con instituciones de educación superior e investigación en Brasil y Portugal, a través de las universidades abiertas de ambos países y de las instituciones miembros para cualificar cursos de educación a distancia. De momento, en esta cooperación se utilizan dos proyectos gratuitos de la Fundación CAPES: EduCAPES y Repositório Aberto, que son plataformas digitales con acceso universal. Además, se imparten clases de docentes brasileños y portugueses en la plataforma UAB Brasil Virtual Learning Environment (AVA), también utilizada por estudiantes de la UAB en Portugal (InfoCAPES, 2019).

Para lograr educación virtual exitosa en la CPLP, las autoridades y expertos han tenido en cuenta los principales elementos que la conforman: el modelo pedagógico, la tecnología apropiada y el papel los actores en el proceso. En este contexto, se deben aprovechen todas sus posibilidades, ya que los cambios que producen en los procesos de enseñanza y aprendizaje, generan la necesidad de disponer de un modelo capaz de dar respuesta a los requerimientos educativos, que surgen como consecuencia de los cambios de paradigmas. Y se están planificando otros proyectos, teniendo en cuenta las posibilidades y perspectivas para expandir y profundizar el conocimiento sobre los acuerdos de cooperación internacional, los supuestos para el éxito de experiencias educativas internacionales y la superación de los desafíos en educación a distancia.

\section{CONCLUSIÓN}

El alto nivel de demanda en la educación a distancia debe ser acompañado de políticas gubernamentales adecuadas, por lo que algunos gobiernos de muchas regiones del mundo se han dado cuenta de las bondades de los sistemas de educación a distancia,

Rev. Nova Paideia - Revista Interdisciplinar em Educação e Pesquisa Brasília/DF, v. 2, n. 1. p. 35 - 46 - ANO 2020 
introduciendo programas de fomento de uso de las TIC en la educación y en otros ámbitos de la sociedad y fomentando nuevas universidades abiertas.

La CPLP está realizando actividades para impulsar la cooperación multinacional en el campo de la educación en general, y de la educación a distancia en particular, con el objetivo de responder a los intereses, prioridades y necesidades de los Estados miembros. Los resultados exitosos de proyectos de cooperación en educación a distancia y las ventajas de una cooperación concertada, han llevado a los expertos y autoridades de la organización a definir políticas y proyectos comunes. En ese sentido, la creación de la Asociación de Educación a Distancia de Países de Lengua Portuguesa, que proporciona a sus miembros productos elaborados por las instituciones educativas más antiguas y de mayor experiencia, y fomenta el diseño e implantación de futuros proyectos.

Asimismo, se observa que el alcance de la educación en este proceso es limitado, por lo que el enfoque de la CPLP hacia la sociedad civil se ha promovido a través de la Comisión Temática sobre Educación, Educación Superior, Ciencia y Tecnología de los Observadores Consultivos de la CPLP, señalando la importancia de llevar a cabo acciones conjuntas con la sociedad, para promover y estimular un desarrollo económico - social que pueda proporcionar una mayor igualdad y justicia social.

\section{REFERENCIAS}

Asociación de Educación a Distancia de Países de Lengua Portuguesa, https://sites.uab.pt/aead/.

Bolaños-Mejías, C., Bueno de la Fuente, G. Alves, F. M. M. (2013). O protagonismo da informação-documentação na cooperação ao desenvolvimento: os recursos educativos abertos nos processos de e-learning. Informação \& Sociedade, 23 (1), pp. 105-115. Disponible en https://periodicos.ufpb.br/ojs/index.php/ies/article/view/15120/9269. Aceso el 31 mayo 2020.

Comunidade dos Países de Língua Portuguesa. Estatutos da Comunidade dos Países de Língua Portuguesa (com revisões de São Tomé/2001, Brasília/2002, Luanda/2005, Bissau/2006 e Lisboa/2007). Disponible en http://www.cplp.org/Files/Filer/Documentos\%20Essenciais/Estatutos CPLP REVLIS0 7.pdf. Aceso el 31 mayo 2020.

Comunidade dos Países de Língua Portuguesa. Universidades vão criar Associação de Educação a Distância. Notícia 03/05/2018. Disponible en https://www.cplp.org/id4447.aspx?Action=1\&NewsId=5743\&M=NewsV2\&PID=10872. Aceso el 31 mayo 2020.

Coordenação de Aperfeiçoamento de Pessoal de Nível Superior (2019). Educação a distância nos Países de Língua Portuguesa. InfoCAPES, 13. Disponible en https://www.capes.gov.br/infocapes/013-novembro-2019/. Aceso el 31 mayo 2020.

Rev. Nova Paideia - Revista Interdisciplinar em Educação e Pesquisa Brasília/DF, v. 2, n. 1. p. 35 - 46 - ANO 2020 
García Aretio, L. (1999). Historia de la educación a distancia. Revista iberoamericana de educación a distancia, 2 (1), 9-27. Disponible en http://revistas.uned.es/index.php/ried/article/view/2084. Aceso el 31 mayo 2020.

Moreiro-González, J. A. (2018). Actuación profesional en ambientes digitales y formación en Ciencia de la Información. Convergências em Ciência da Informação, São Cristóvão SE, V. $1, \quad \mathrm{n}$ 1, pp. 03-25. Disponible en https://seer.ufs.br/index.php/conci/article/view/9344. Aceso el 31 mayo 2020.

Naciones Unidas (2015). Agenda 2030 sobre el Desarrollo Sostenible. Disponible en https://www.un.org/sustainabledevelopment/es/. Aceso el 31 mayo 2020. Aceso el 31 mayo 2020.

Rojas Machado, N., Pérez Clemente, F., Torres Milord, I., \& Peláez Gómez, E. (2014). Las aulas virtuales: una opción para el desarrollo de la Educación Médica. Edumecentro, 6 (2), pp. 231-247. $\quad$ Disponible http://scielo.sld.cu/scielo.php?script=sci arttext\&pid=S2077-28742014000200016. Aceso el 31 mayo 2020.

Salinas, J. (2004). Hacia un modelo de educación flexible: Elementos y reflexiones. Madrid. Pearson-Prentice Hall.

Souza, J. A. G., Segenreic, S. C. (2018). Expansão da educação superior e internacionalização horizontal via Brasi/UAB: uma visão de Moçambique como parceira do Acordo Bilateral. Anais XV Congresso Brasileiro de Ensino Superior a Distância (ESUD) e o IV Congresso Internacional de Educação Superior a Distância (CIESUD). Disponible en https://esud2018.ufrn.br/wp-content/uploads/187304 1 ok.pdf. Aceso el 31 mayo 2020.

Souza, J. A. G., Segenreic, S. C. (2018). O Programa Brasil/UAB-Moçambique: política pública e/ou Prpgrama de cooperação educacional. Anais CIET: EnPED:2018. Educação e Tecnologias: Gestão e política. Disponible en https://cietenped.ufscar.br/submissao/index.php/2018/article/view/74. Aceso el 31 mayo 2020.

UNESCO (2002). Aprendizaje abierto y a distancia. Consideraciones sobre tendencias, políticas $y \quad$ estrategias. Montevideo. Trilce. Disponible en https://www.recursos.portaleducoas.org/politicas-informes/aprendizaje-abierto-ydistancia-consideraciones-sobre-tendencias-pol-ticas-y. Aceso el 31 mayo 2020.

UNESCO (2006). Division of Higher Education Section for Reform, Innovation and Quality Assurance (RIQ). UNESCO/OECD Guidelines on Quality Provision In CrossBorder Higher Education, Drafting Meeting 3. UNESCO"s Capacity Building Activities In Qualifications Recognition, Quality Assurance And Accreditation: Towards A Coherent

Rev. Nova Paideia - Revista Interdisciplinar em Educação e Pesquisa Brasília/DF, v. 2, n. 1. p. 35 - 46 - ANO 2020 
Framework. Paris, pp. 17-18. Disponible en http://www.oecd.org/education/skillsbeyond-school/34266472.pdf. Aceso el 31 mayo 2020.

Universidade Aberta, https://portal.uab.pt.

United Nations (1998). World Conference on Higher Education in the Twenty-first Century: Vision and Action. Paris. Disponible en https://unesdoc.unesco.org/ark:/48223/pf0000113779. Aceso el 31 mayo 2020. 